\title{
Anticoagulation for Cardioversion of Atrial Fibrillation
}

\author{
David M. Weinberg, MD, and G.B. John Mancini, MD
}

A trial fibrillation (AF) is a common arrhythmia associated with a broad spectrum of underlying diseases that include systemic hypertension, rheumatic heart disease and coronary artery disease. ${ }^{1}$ Several studies have documented the strong relation between chronic $\mathrm{AF}$ and emboli. $^{2,3}$ In addition, an increased risk of embolism exists in the setting of cardioversion of AF to sinus rhythm. ${ }^{4,5}$ In the best study to date, Bjerkelund and Orning ${ }^{6}$ reported on 572 attempted cardioversions in 437 patients and observed a $0.8 \%$ incidence of embolization in long-term anticoagulated patients compared with $5.3 \%$ in a nonanticoagulated group. Shortcomings of this study included lack of randomization, no evaluation of shortterm therapy and inclusion of arrhythmias such as atrial flutter and atrial tachycardia. Based on such work, current recommendations include anticoagulation for 2 to 4 weeks before cardioversion to allow adherence and endothelialization of existing thrombus and 1 to 4 weeks after cardioversion to provide coverage for late resumption of atrial activity. ${ }^{7,8}$ The present report evaluates these recommendations in light of our experience over the past 10 years.

The University of Michigan Hospital's Medical Information Department identified 150 patients with $A F$ who underwent cardioversion over the study decade. After 105 patient charts were located from that total, 79 palients were deemed appropriate for siudy and 26 were excluded. Exclusion criteria included rhythm disturhances other than $A F$ and transient episodes of $A F$ during cardiopulmonary arrest or after cardiac surgery ( $<24$ hours). A cerebral embolism was considered present and related to the cardioversion if a circumscribed focal deficit developed within 2 weeks of cardioversion. ${ }^{9}$ All events occurred, however, within 72 hours.

Most patients were men (57\%) and a significant percentage had underlying organic disease (see later). Age ranged from 16 to 84 years. Approximately $14 \%$ (11 patients) had a history of prior stroke or transient ischemic attack. Of those, a third (4 of 11) experienced an embolic event at the time of presentation with new onset of $A F$. Approximately $10 \%$ of patients had either clinical (elevated jugular venous pressure, $S_{3}$, rales) or radiologic evidence of congestive heart failure. One half of the study population received digoxin before cardioversion. Bundle branch block was found in 10 (13\%) patients.

Duration of $A F$ ranged from $<48$ hours to $>12$ months. It lasted <48 hours in $19(24 \%)$ patients, 48 hours to 2 weeks in $16(20 \%)$, from 2 weeks to 6 months in $24(30 \%)$ and $>6$ months in $18(23 \%)$.

From the Department of Intcrnal Medicine, Division of Cardiology, the University of Michigan and Veterans Administration Medical Center, Ann Arbor, Michigan 48105. Manuscript received August 23, 1988; revised manuscript received December 16, 1988, and accepted December 19.
Echocardiograms were performed in 52 of the 79 patients. Approximately $62 \%$ (32 of 52) were found to have left atrial enlargement $(>40 \mathrm{~mm})$. Almost a third had mitral (10 of 52) or aortic ( 6 of 52) valvular abnormalities. Left ventricular dysfunction was found in $31 \%$ (16 of 52) of these patients. Radionuclide angiograms were available in $38 \%$ of the study population and ejection fractions $<45 \%$ were documented in 18 patients. The majority (93\%) was electrically cardioverted $(7 \%$ pharmacologically). Successful conversion to normal sinus rhythm was accomplished in $84 \%$ of the patients. The duration of sinus rhythm ranged from 12 hours to $>5$ years.

Sixty-two percent of patients who did not experience cardioversion-related emboli were anticoagulated before cardioversion, most for $<1$ month. A relatively small number ( 8 of 48 ) of those who did not experience an embolic event received long-term anticoagulation $(>1$ year). Criteria for therapeutic anticoagulation included a prothrombin time 1.5 to 2.0 times the control values. Prothrombin times (by rabbit brain thromboplastin) were obtained within 72 hours of cardioversion in all patients receiving anticoagulation therapy. Prothrombin times were obtained randomly in the 28 patients who did not receive anticoagulation.

None of the anticoagulated patients had a cardioversion-related stroke. In contrast, $7 \%$ (2 of 28) who were not anticoagulated or who received $<2$ days of heparin therapy ("nonanticoagulated group") had an embolic. complication. By studying the patients who experienced a stroke, a "high-risk" subgroup emerged, comprised of patients $>55$ years who had coronary heart disease, cardiomyopathy or hypertension or both, and duration of $A F>1$ year. Fifty percent of the nonanticoagulated patients in this subgroup had an embolic complication. Five others fitting this subgroup definition who received short-term anticoagulation had no complications.

Hemorrhagic complications were seen in 8 patients (10\%). One patient had only minimal bruising. Seven other bleeding events were noted per 16,440 anticoagulated patient days ( 0.16 events/patient-year). Specific complications included hematuria (2 patients), epistaxis (2), guaiac positive stool (1), menorrhagia (1) and diverticular gastrointestinal bleeding (1). Among the 51 patients who were anticoagulated, $14 \%$ experienced a bleeding complication. No hemorrhagic events occurred in the 28 patients not anticoagulated. Of note, no bleeding events were seen among those patients who received short-term ( $<1$ month) anticoagulation therapy. Hemorrhagic complications occurred almost exclusively in the subset of patients receiving long-term anticoagula. tion, often necessitated by the presence of prosthetic valves. There was no correlation between blecding complications and time of cardioversion. No markedly ele- 
vated prothrombin times were noted during any hemorrhagic event.

Of the study population overall, 2 patients had pericardioversion-related embolus (equal to $7 \%$ of the nonanticoagulated cohort). Both had cardiomegaly, longduration AF ( $>1$ year) and were either receiving no anticoagulants or had $<2$ days of heparin therapy. In contrast, no embolic events were observed in the group of patients therapeutically anticoagulated. Patients with underlying organic heart disease and long-standing AF are least likely to be successfully cardioverted and maintained in sinus rhythm, ${ }^{10}$ although in this instance both patients were successfully cardioverted.

The risks of anticoagulation provide strong motivation to forego this prophylactic therapy in many patients requiring cardioversion. Whereas $14 \%$ of anticoagulated patients in this study experienced a bleeding episode, only $2 \%$ were life-threatening. Of importance is the fact that no bleeding complications occurred among patients receiving short-term ( $<1$ month) anticoagulation in this study. Well-managed, short-term anticoagulation of selected patients appears to be a beneficial prophylactic maneuver, with little risk of significant hemorrhagic sequelae. Given the small sample size, this conclusion is still presumptive. A large, controlled study would be desirable to more definitively assess the utility of anticoagulation in this common clinical situation. In the interim, our experience supports consideration of short-term anticoagulation when cardioversion of $\mathrm{AF}$ is indicated.

1. Kannel WB, Abbott RD, Savage DD, McNamara PM. Epidemiologic features of chronic atrial fibrillation. The Framingham Study. $N$ Engl J Med 1982; 306:1018-1022.

2. Wolf PA, Kannel WB, McGee DL, Meeks SL, Bharucha NE, McNamara PM. Duration of atrial fibrillation and imminence of stroke: the Framingham Study. Stroke 1983;14:664-667.

3. Sherman DG, Goldman L, Whiting RB, Jurgensen K, Kaste M, Easton JD. Thromboembolism in patients with atrial fibrillation. Arch Neurol 1984:41:708710.

4. Selzer A, Kelly JJ, Johnson RB, Kerth WJ. Immediate and long-term results of electrical conversion of arrhythmias. Prog Cardovasc Dis 1966:9:90-104.

5. McCarthy C, Varghese PJ, Barritt DW. Prognosis of atrial arrhythmias treated by electrical counter shock therapy: a three year follow-up $\mathrm{Br}$ Heart $\mathrm{J}$ 1969;31:496-500

6. Bjerkelund $\mathrm{CJ}$, Oning $\mathrm{OM}$. The efficacy of anticoagulant therapy in preventing embolism related to D.C. electrical cardioversion of atrial fibrillation. $A m \mathrm{~J}$ Cardiol 1969;23.208-215.

7. De Silva RA, Graboys TB, Podrid PJ, Lown B. Cardioversion and defibrillation Am Heart J 1980:100:881-895.

8. Mancini GBJ, Goldberger AL. Cardioversion of atrial fibrillation. consideration of embolization, anticoagulation, prophylactic pacemaker, and long-term success. Am Heart J 1982;104.617-621.

9. Rowland LP. Merritt's Textbook of Neurology. Seventh ed. Philadephia: Lea \& Febiger, 1984:170.

10. Morris JJ Jr, Peter RH, McIntosh HD. Electrical conversion of atrial fibrillation. Immediate and long-term results and selection of patients. Ann Intern Med $1966,65: 216-231$.

\title{
Electrophysiologic Effects, Efficacy and Tolerance of Class Ic Antiarrhythmic Agents in Wolff-Parkinson-White Syndrome
}

\author{
Antonis S. Manolis, MD, Deeb N. Salem, MD, and N.A. Mark Estes, III, MD
}

A fter extensive evaluation in clinical investigation, the class Ic antiarrhythmic agents flecainide and encainide have recently been approved for the treatment of ventricular arrhythmias. Preliminary reports indicate that these drugs may be useful in the treatment of a variety of supraventricular tachycardias, including atrioventricular reciprocating tachycardia and atrial fibrillation in the setting of Wolff-Parkinson-White (WPW) syndrome. ${ }^{1-5}$ This prospective study was undertaken to evaluate systematically the electrophysiologic effects, clinical efficacy and tolerance of flecainide and encainide in patients with the WPW syndrome.

Eighteen consecutive patients including 14 men and 4 women, aged $41 \pm 18$ years (range 20 to 72 ), with symptomatic preexcitation syndrome were studied before and after oral flecainide or encainide. In 1 patient a concealed bypass tract was present, while in the remaining 17 preexcitation was manifest. Presenting symptoms included palpitations in 3 patients, presyncope in 9 patients and syncope in 6 patients. The presenting clinical arrhythmia was reciprocating tachycardia in 10 patients

From the Cardiac Arrhythmia Service, Division of Cardiology, Department of Medicine, Tufts University School of Medicine, 750 Washington Street, Boston, Massachusetts 02111. Manuscript received October 31, 1988; revised manuscript received and accepted December 12, 1988. and atrial fibrillation in 8 patients. In 2 patients atrial fibrillation degenerated into ventricular fibrillation. The average number of previous unsuccessful drug trials before referral for electrophysiologic study was $1.9 \pm 1.0$ (range 0 to 4 ).

All patients underwent baseline electrophysiologic study in the drug-free state. The programmed electrical stimulation was performed using standard techniques. ${ }^{4-6}$ Atrial fibrillation was initiated in all patients by rapid burst atrial pacing. After the baseline study, 12 patients received oral flecainide in dosages of 100 to 200 $\mathrm{mg}$ twice daily for 4 days (mean daily dose $250 \pm 76 \mathrm{mg}$ ) and 5 patients received oral encainide in doses of 25 to 50 $m g$ thrice daily for 3 days (mean daily dose $108 \pm 24$ $m g$ ) before follow-up evaluation. One patient initially placed on encainide had an increase in the frequency of reciprocating tachycardia occurrences without prolongation of the retrograde refractory period of the bypass tract. This patient was switched from encainide to flecainide with improved clinical and electrophysiologic results. Serum levels of encainide and its metabolites were not obtained at follow-up studies. Flecainide serum levels averaged $0.6 \pm 0.2 \mathrm{mg} /$ liter (range 0.4 to 0.9 ).

All patients were followed clinically at the Cardiac Arrhythmia Clinic at 3-month intervals as well as by 